\title{
Una reina en estado: Leonor de Castilla (1241-1290). Perspectiva historiográfica y aproximación a su estudio ${ }^{1}$
}

\author{
A pregnant queen: Eleanor of Castile (1241-1290). Overview and \\ approximation to its study
}

\author{
ANDREA BERGAZ ÁLVAREZ \\ Universidad Complutense de Madrid \\ anberg01@ucm.es
}

Resumen: Este artículo pretende llevar a cabo un primer estado de la cuestión en español sobre los embarazos y partos de una reina castellana de Inglaterra: Leonor de Castilla (1240-1291). En primer lugar, se presenta al personaje, remarcándose la importancia que tuvieron la maternidad y su rol como "proveedora de herederos" en su vida política y en su recuerdo. Sorprendentemente - aunque en consonancia con los estudios historiográficos generales sobre el personaje - , los trabajos sobre la primera etapa de la maternidad de esta reina se revelan escasos, limitados y con carencias, algo en lo que sin duda han influido las dificultades que plantean las fuentes primarias directas para el conocimiento del personaje. En este artículo se presentan algunas fuentes generales que otros historiadores han manejado desde diversas perspectivas por sus posibilidades y potencial, valorándose de qué modo podrían ayudar al conocimiento de la reginalidad de Leonor de Castilla.

Palabras clave: Leonor de Castilla, embarazo, reginalidad, historiografía, Inglaterra.

Abstract: This article aims to provide a first overview in Spanish of the childbearing of a Castilian queen of England: Eleanor of Castile (1240-1291). Firstly, the queen is presented, highlighting the importance that motherhood and her role as "heirs' provider" played in her political life and in her memory. Surprisingly -although accordingly with the general historiographical studies about this character- the works about the first stage of the Elanor's maternity are scarce, limited and they present mistakes, something which has undoubtedly been influenced by the difficulties posed by the direct primary sources about this queen. This article presents some general sources that other historians have used from different perspectives because of their possibilities and potential, assessing how they could help in the knowledge because of the queenship of Eleanor of Castile.

Keywords: Eleanor of Castile, pregnancy, queenship, historiography, England.

\footnotetext{
${ }^{1}$ Este artículo ha sido elaborado a partir de la ponencia presentada en el I Congreso de Jóvenes Investigadores JIMENA Creciendo en el Medievo, celebrado en la Universidad Complutense de Madrid los días 18, 19 y 20 de noviembre de 2019. Agradezco enormemente al profesor Martín Alvira Cabrer sus observaciones y consejos, sin los cuales este artículo no habría sido posible. Me gustaría también agradecer a Iván Curto Adrados su ayuda en la preparación de la ponencia y a los revisores anónimos sus valiosas recomendaciones.
}

Recibido: 11 de diciembre de 2020; aceptado: 20 de septiembre de 2021; publicado: 30 de septiembre de 2021. Revista Historia Autónoma, 19 (2021), pp. 125-135

e-ISSN: 2254-8726; https://doi.org/10.15366/rha2021.19.006 


\section{Introducción}

Leonor de Castilla (1241-1290), hija del rey de Castilla y León Fernando III el Santo y de su segunda esposa Juana de Ponthieu, fue reina de Inglaterra en virtud de su matrimonio con Eduardo I de Inglaterra desde 1272 hasta su muerte en 1290. Pese al auge de los estudios sobre la reginalidad (queenship) ${ }^{2}$, y aun tratándose de la esposa de un rey crucial para la historia de Inglaterra, su figura no ha recibido demasiada atención por parte de la historiografía anglosajona, menos aún de la española. Son escasos los estudios académicos sobre el personaje, la mayor parte de ellos centrados en la cuestión financiera o patrimonial y en el programa funerario desarrollado tras su muerte. A ello se suma la existencia de una considerable cantidad de obras de divulgación que, en muchos casos, contienen errores, anacronismos o imprecisiones que han contribuido a alimentar los mitos y tópicos que siguen envolviendo el recuerdo y el estudio de esta reina ${ }^{3}$. La consecuencia de todo ello es que, como bien indicaba John C. Parsons en los años ochenta, con Leonor de Castilla nos encontramos todavía frente a un "historical and historiographical enigma"4.

No obstante, hay que destacar algunas contribuciones académicas importantes para el estudio de este personaje, empezando por las del propio Parsons, pionero en el estudio de la reginalidad, que ha dedicado gran parte de su carrera a explorar de forma rigurosa el papel

\footnotetext{
${ }^{2}$ Entre las obras clásicas sobre este tipo de estudios hay destacar, entre otras: Earenfight, Theresa, Queenship in Medieval Europe, Nueva York, Palgrave Macmillan, 2013. https://doi.org/10.1007/978-1-137-30392-9; Parsons, John C. (ed.), Medieval Queenship, Stroud, Sutton, 1998. https://doi.org/10.1007/978-1-137-08859-8 9 o Woodacre, Elena (ed.), Queenship in the Mediterranean. Negotiating the Role of the Queen in the Medieval and Early Modern Eras, Basingstoke, Palgrave Macmillan, 2013. https://doi.org/10.1057/9781137362834 4.

Empleamos el término "reginalidad" de acuerdo con la definición y traducción propuesta por Nuria SillerasFernández ("Queenship en la Corona de Aragón en la Baja Edad Media: estudio y propuesta terminológica", La Corónica. A Journal of Medieval Hispanic Languages, Literatures and Cultures, 32 (2003), p. 132. https:// doi.org/10.1353/cor.2003.0018). Se trata de un vocablo cada vez más empleado en el ámbito académico hispano por su relación con la palabra catalana "reginal", que sí aparece en la documentación medieval (Pelaz Flores, Diana y Val Valdivieso, María Isabel del, "La historia de las mujeres en el siglo XXI a través del estudio de la reginalidad medieval", en Revista de Historiografía, 22 (2017), p. 107). Aunque no es un término aceptado por la RAE y la traducción plantea algunos problemas, recurrimos a él siguiendo las tendencias historiográficas actuales, tanto española como internacional. No obstante, conviene señalar que las resistencias al empleo de este vocablo no se limitan a lo terminológico. Así lo demuestra una de las obras de María Jesús Fuente Pérez (Violante de Aragón, reina de Castilla, Madrid, Dykinson, 2017), en la que se muestra reacia a la creación de moldes o categorías comunes aplicables a reinas de diferentes partes de Europa. La autora defendió también esta idea en su comunicación "Usos y abusos de términos y conceptos", Nombrar para comprender. Conceptos y debates para la Historia Medieval peninsular (Casa Árabe de Madrid, 11 de julio de 2019), inédito.

${ }^{3}$ Se dan presentismos peligrosos e importantes como el de Sara Cockerill, cuando hablaba de la reina como una adelantada al movimiento feminista (Eleanor of Castile. The Shadow Queen, Stroud, Amberley Publishing, 2006, p. 19). No obstante, a pesar de sus carencias metodológicas y de su manifiesta parcialidad, esta obra aporta algunas perspectivas novedosas e interesantes. Otras publicaciones con carácter divulgativo son Gentleman, David, $A$ Cross for Queen Eleanor. The Story of the Building of the Medieval Charing Cross, the Subject of the Decorations on the Northern Line Platforms of the new Charing Cross Underground Station. Printed to Mark the Occasion of the Opening of the Jubilee Line by his Royal Highness the Prince of Wales 30 April 1979, Londres, London Transport, 1979; Ybarra, Fernando de, Matrimonios reales hispano-británicos en el Medievo. Póker de reinas, Salamanca, Universidad Pontificia de Salamanca, 1999; y Warrington, Decca, The Eleanor Crosses: The Story of King Edward I's Lost Queen and her Architectural Legacy, Oxford, Signal Books, 2018.

${ }^{4}$ Parsons, John C., Eleanor of Castile: Queen and Society in Thirteenth-Century England, Basingstoke, Macmillan, 1998, p. xi.
} 
político de Leonor de Castilla. De hecho, a él se deben la mayor parte de aportaciones académicas referidas a esta reina 5 . Sobre la cuestión del patrimonio y los recursos de Leonor de Castilla, también trabajada por Parsons, destaca especialmente Hilda Johnstone ${ }^{6}$. Existen varios estudios académicos sobre su actividad cultural, especialmente en relación con su programa funerario, si bien es cierto que centrados en su mayoría en la cuestión artística, de modo que interesan poco a nuestro tema y no los trataremos en este artículo?.

Así, el estudio de la maternidad, los embarazos y partos de la reina Leonor de Castilla ofrece aún muchas posibilidades. Estamos ante una cuestión en gran medida inexplorada en el estudio de este personaje, si bien es cierto que Parsons ha investigado sobre ello - y no únicamente en referencia a esta reina- en algunos de sus trabajos ${ }^{8}$. Por otro lado, el impulso de los estudios de reginalidad y de la llamada "Nueva Historia Política" ha llevado a algunos historiadores a indagar sobre el papel de la maternidad en el ejercicio del poder femenino durante el Medievo9. La mayor parte de estos estudios se han centrado en analizar los casos en los que la maternidad constituyó un instrumento legitimador y un revulsivo para el poder

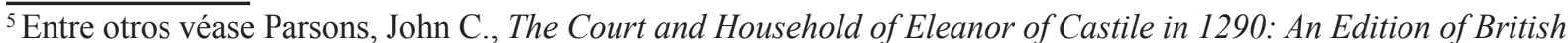
Library, Additional Manuscript 35294, Toronto, Pontifical Institute of Mediaeval Studies, 1977; Parsons, John C., "Eleanor of Castile and the Countess Margaret of Ulster", Genealogists'Magazine, 20 (1984), pp. 335-340; Parsons, John C., "Piety, Power and Reputation of Two Thirteenth-Century English Queens", en Vann, Theresa, Queens, Regents and Potentates, Dallas: Academia, 1993, pp. 107-124; y Parsons, John C., Eleanor of Castile... op. cit. ${ }^{6}$ Johnstone, Hilda, "The Queen's Household", en Williard, James y Morris, William (eds.), The English Government at Work 1327-1336, Cambridge, The Mediaeval Academy of America, 1947, pp. 250-299; y Johnstone, Hilda, “The Queen's Exchequer under the Three Edwards", en Edwards, John et al. (eds.), Historical Essays in Honour of James Tait, Manchester, Manchester University Press, 1993, pp. 143-153.

${ }^{7}$ Entre otros, véase Hallam, Elizabeth, "Introduction: The Eleanor Crosses and Royal Burial Customs", en Parsons, David (ed.), Eleanor of Castile 1290-1990: Essays to Commemorate the 700 th Anniversary of her Death: 28 November 1290, Stamford, University of Leicester, 1991, pp. 9-22; Tolley, Thomas, "Eleanor of Castile and the Spanish Style in England", en Ormrod, Mark, England in the Thirteenth Century: Proceedings of the 1989 Harlaxton Symposium (1991), Stamford, Watkins, 1991, pp. 167-192; Coldstream, Nicola, "The Tomb of Queen Eleanor in Westminster Abbey: An Evaluation of the Documentary Evidence", en Beck, Herbert et al. (eds.), Studien zur Geschichte der Europänischen Skulptur im 12./13. Jahrhundert. Bd. 1, Text, Frankfurt, Herich Verlag, 1994, pp. 101-108; Parsons, John C., "Never was a Body Buried in England with such Solemnity and Honour: the Burials and Posthumous Commemorations of English Queens to 1500", en Duggan, Anne (ed.), Queens and Queenship in Medieval Europe: Proceedings of a Conference held at King's College London, April 1995, Woodbridge, Boydell, 1997, pp. 317-337; Walker, Rose, "Leonor of England and Eleanor of Castile: Anglo-Iberian Marriage and Cultural Exchange in the $12^{\text {th }}$ and $13^{\text {th }}$ Centuries", en Bullón-Fernández, María (ed.), England and Iberia in the Middle Ages, $12^{\text {th }}-15^{\text {th }}$, Nueva York, Palgrave Macmillan, 2007, pp. 67-88. https://doi.org/10.1057/9780230603103 5; y Dilba, Carsten, Memoria Reginae: Das Memorialprogramm für Eleonore von Kastilien, Hildesheim, Universität von Bonn, 2009.

${ }^{8}$ Véase Parsons, John C., "The Year of Eleanor of Castile's Birth and her Children by Edward I", Mediaeval Studies, 46 (1984), pp. 245-265. https://doi.org/10.1484/J.MS.2.306316; Parsons, John C., "The Pregnant Queen as Counsellor and the Medieval Construction of Motherhood", en Parsons, John C. y Wheeler, Bonnie (eds.), Medieval Mothering, Nueva York, Garland, 1996, pp. 39-61; y Parsons, John C., "Que nos lactauit in infancia: The Impact of Childhood Caregivers on Plantagenet Family Relationships in the Thirteenth and Early Fourteenth Centuries", en Rousseay, Constance y Rosenthal, Joel, Women, Marriage and Family in Medieval Christendom: Essays in Memory of Michael Sheehan, Kalamazoo, Western Michigan University, 1998, pp. 289-324.

${ }^{9}$ Cañas Gálvez, Francisco de Paula, "Las Casas de Isabel y Juana de Portugal, reinas de Castilla. Organización, dinámica institucional y prosopografía (1447-1496)”, en Martínez Millán, José y Marçal Lourenço, María Paula, Las Relaciones Discretas entre las Monarquías Hispana y Portuguesa: las Casas de las reinas (siglos XV-XIX), Madrid, Polifemo, 2009, p. 11. Véase Parsons, John C. y Wheeler, Bonnie (eds.), Medieval Mothering op. cit.; Segura Graíño, Cristina, "Las parteras: imaginario religioso, realidad social y funcionalidad política", en Cid López, Rosa María (coord.), Maternidades: representaciones y realidad social. Edades Antigua y Media, Madrid, Asociación Cultural Al-Mudayna, 2010, pp. 273-284; y Fuente Pérez María Jesús, "Gracias, nodriza. La estima de la lactancia y la crianza a través del ejemplo medieval", Dilemata, 25 (2017), pp. 55-67. «https://www.dilemata. net/revista/index.php/dilemata/article/view/412000132»" [consultado el 29 de septiembre de 2020].
} 
de las reinas ${ }^{10}$. No obstante, la historiografía comienza a ampliar la perspectiva y a interesarse también por los casos en los que las relaciones maternofiliales no fueron buenas y la maternidad constituyó un obstáculo político para madre e hijos ${ }^{11}$.

En los últimos años se viene también explorando, con un creciente interés, la gestación y el parto como sistema cultural y espacio de poder femenino, especialmente en el ámbito reginal, demostrándose sus enormes posibilidades en varios estudios sobre la Edad Moderna ${ }^{12}$. Las fuentes disponibles para el conocimiento de esta cuestión en la Edad Media son menores, pero consideramos que estudios de caso como el de Leonor de Castilla — con una prolífica descendencia - pueden ser muy valiosos a la hora de avanzar este prometedor campo de estudio. Así pues, en este trabajo nos proponemos dos objetivos: primero, recoger y ordenar la información recopilada hasta ahora por los especialistas sobre los embarazos y partos de Leonor de Castilla; y, a partir de ahí, intentar despertar el interés de la historiografía española por este personaje tan poco conocido, mostrando las posibilidades de estudio que ofrece su figura en general, y el estudio de su maternidad en particular.

\section{Leonor de Castilla, mater et coniunx regis}

Como bien indicó Janet Nelson a finales del pasado siglo, "la reina embarazada era garantía de la supervivencia e integridad del reino, así como de la paz y el control"13. La perpetuación de la dinastía era efectivamente una de las principales funciones de las consortes. La historiografía ha señalado cómo aquellas reinas que por motivos biológicos no podían engendrar se veían en la necesidad de explotar en mayor medida otras formas de legitimación que también eran

\footnotetext{
${ }^{10}$ En este sentido caben destacar los estudios sobre reinas que promovieron y defendieron los derechos de sus hijos al trono, como, por ejemplo, Berenguela de Castilla. Véase, entre otros, Bianchini, Janna, The Queen's Hand: Power and Authority in the Reign of Berenguela of Castile, Filadelfia, Pennsylvania Press, 2012.

${ }^{11}$ Véanse al respecto, García-Fernández, Miguel, "Doña Teresa y doña Urraca: la figura de la madre como obstáculo político a comienzos del siglo XII”, en Gallego Franco, Henar y García Herrero, María del Carmen (coords.), Autoridad, poder e influencia: mujeres que hacen Historia, Barcelona, Icaria Editorial, 2017, pp. 115130 y Pallares Méndez, María del Carmen, "Urraca de León y su familia. La parentela como obstáculo político", en Trillo San José, María del Carmen (coord.), Mujeres, familia y linaje en la Edad Media, Granada, Universidad de Granada, 2004, pp. 69-104.

${ }^{12}$ Como el dossier monográfico "Nacer en tiempos de Calderón: universos femeninos y culturas del parto en el Siglo de Oro", Revista Historia Autónoma, 12 (2020), pp. 13-122. «https://revistas.uam.es/historiaautonoma/issue/ view/rha2019.16» [consultado el 29 de septiembre de 2020]. Entre otros trabajos, véase también Carlos Varona, María Cruz de, "Entre el riesgo y la necesidad: embarazo, alumbramiento y culto a la Virgen en los espacios femeninos del Alcázar de Madrid (siglo XVII)", Arenal. Revista de Historia de mujeres, 13 (2006), pp. 263-290. «https://revistaseug.ugr.es/index.php/arenal/article/view/2999» [consultado el 29 de septiembre de 2020]; Carlos Varona, María Cruz de, Nacer en palacio. El ritual del nacimiento en la corte de los Austrias, Madrid, Centro de Estudios Europa Hispánica, 2018; y Hannah Fischer-Monzón, "Nacer en tiempos de Calderón. Lucina, Diana y (la) Luna, las diosas lunares del parto en el Siglo de Oro", en Memoria y civilización: anuario de Historia, 21 (2018), pp. 61-88. https://doi.org/10.15581/001.21.031

${ }^{13}$ Nelson, Janet, "Medieval Queenship", en Mitchell, Linda (ed.), Women in Medieval Western European Culture, Nueva York, Routledge, 1999, pp. 179-207.
} 
importantes para aquellas que engendraban herederos. Estas se apoyaban fundamentalmente en su papel religioso, convirtiéndose en modelos de castidad y de piedad ${ }^{14}$.

Durante el siglo XIII, tras lo que algunos han considerado un resurgir de la reginalidad inglesa en el siglo XII bajo unos nuevos parámetros ${ }^{15}$, esta función se convirtió, junto con la intercesión, en la base de la posición política de las reinas consortes ${ }^{16}$. Ambas facetas habitualmente se conjugaban. Así ocurría en el ordo de la coronación, en el que se relacionaba a la nueva reina con la Virgen María y la Esther bíblica — personajes que encarnaban dichas funciones - ${ }^{17}$. Textos de la época y de principios del siglo XIV, como este acto de intercesión de la reina Felipa de Hainaut (esposa de Eduardo III) narrado por Jean Froissart, demuestran la conexión entre maternidad e intercesión: Adont fist la noble royne d'Engleterre grant humilité, qui estoit durement enchainte, et ploroir si tenremet de pité, que on ne le pooit soustenir ${ }^{18}$.

En el caso de Leonor de Castilla, aunque la importancia de la función intercesora plantea dudas, parece más claro que la maternidad desempeñó un papel crucial en su reginalidad y en su posición política ${ }^{19}$. La magnitud de su descendencia excedió las expectativas de una consorte. Así, junto a la buena relación y la intimidad que compartía con Eduardo ${ }^{20}$, su papel como "proveedora de herederos" constituyó su principal fuente de influencia política y una de las facetas más relevantes de su ejercicio del poder. Su condición de "madre prolífica" condicionó su vida y también su recuerdo. Ese rol maternal ha alimentado el mito que desde finales de la Edad Media rodea su persona y que, en gran medida, continúa vivo hoy en día ${ }^{21}$. Leonor ha

\footnotetext{
${ }^{14}$ Earenfight, Theresa, Queenship in Medieval... op. cit., p. 8. Sobre reinas sin hijos y la conservación del poder e influencia véase, Earenfight, Theresa, "Queens with Child and Without", en Queens in the Middle Ages, 2014 [en línea], https://theresaearenfight.com/2014/08/18/queens-with-child-and-without/

${ }^{15} \mathrm{El}$ debate acerca de los límites cronológicos de la reginalidad en Bennet, Judith, History Matters. Patriarchy and the Challenge of Feminism, Filadelfia, University of Pennsylvania Press, 2006, pp. 54-81; y Earenfight, Theresa, "Medieval Queenship", en History Compass, 15/3 (2017), p. 5. https://doi.org/10.1111/hic3.12372

${ }^{16}$ Para una visión global sobre la reginalidad inglesa de los siglos XI-XIII, véase Parsons, John C., "Queen's Intercession in Thirteenth-Century England", en Carpenter, Jennifer y McLean, Sally-Beth (eds.), The Power of the Weak Studies on Medieval Women, Urbana, University of Illinois Press, 1995, pp. 147-177; y Stafford, Pauline, "The Portrayal of Royal Women in England, Mid-Tenth to Mid-Twelfth Centuries", en Parsons, John C. (ed.), Medieval Queenship... op. cit., pp. 143-167.

${ }^{17}$ Parsons, John C., "The Pregnant Queen... op. cit., pp. 42-43.

${ }^{18}$ Froissart, Jean, Oeuvres, editado por Kervyn de Lettenhove, Bruselas, 1868, vol. 5, p. 205. En Parsons, John C., "The Pregnant Queen... op. cit., p. 40: "Then the noble queen of England, who was heavily pregnant, humbled herself greatly and wept so tenderly from pity that none could bear it".

${ }^{19}$ Sobre la cuestión y el debate en torno a la intercesión, véase Parsons, John C., The Court... op. cit., p. 8; Parsons, John C., "Ritual and Symbol in the English Queenship to 1500", en Fradenburg, Louise (ed.), Women and Sovereignty, Edimburgo, Edimburgh University Press, 1992, p. 66; Parsons, John C., "Piety, Power... op. cit., p. 123; Parsons, John C., "Queen's Intercession... op. cit., p. 152; Parsons, John C., "The Pregnant Queen... op. cit., pp. 46 y 53-54; Parsons, John C., "Never was a Body... op. cit., pp. 332-333; Parsons, John C., Eleanor of Castile... op. cit., pp. 37, 153-154 y 248; y Cockerill, Sara, Eleanor of Castile... op. cit., pp. 218-222 y 254-255. ${ }^{20}$ Sobre el impacto político de la intimidad y complicidad personal entre rey y reina véase Woodacre, Elena, The Queens Regnant of Navarre: Succession, Politics and Partnership, 1274-1512, Nueva York, Palgrave Macmillan, 2013. https://doi.org/10.1057/9781137339157; Katz, Melissa, "The Final Testament of Violante de Aragón (c. 1236-1300/01): Agency and (Dis)empowerment of a Dowager Queen", en Woodacre, Elena (ed.), Queenship in the Mediterranean... op. cit., pp. 51-71; y Woodacre, Elena, "Ruling \& Relationships: The Fundamental Basis of the Exercise of Power? The Impact of Marital \& Family Relationships on the Reigns of the Queens Regnant of Navarre (1274-1517)", en Anuario de Estudios Medievales, 46, 1 (2016), pp. 167-201, https://doi:10.3989/ aem.2016.46.1.05. Dicha buena relación se aprecia en los brevísimos periodos de separación entre ambos, lo que, a su vez, permite explicar el elevado y excepcional número de hijos de la pareja.

${ }^{21}$ La mitificación de Leonor de Castilla en Parsons, John C., "Legend and Reality Through Seven Centuries", en Parsons, David (ed.), Eleanor of Castile... op. cit., pp. 23-54; y Parsons, John C., Eleanor of Castile... op. cit., pp. 205-254.
} 
pasado a la historia como una de las reinas inglesas más fecundas (algunos autores hablan de hasta dieciséis hijos) y, como bien indica Parsons, no es casualidad que, al igual que la citada Felipa de Hainaut (madre de trece hijos), haya disfrutado de una muy buena reputación durante los siglos XIX y $\mathrm{XX}^{22}$.

El personaje de Leonor de Castilla es por lo tanto un interesante y relevante sujeto de estudio en el campo de la maternidad reginal. No obstante, la historiografía ha tratado muy poco la cuestión de sus embarazos y partos. Las razones son múltiples, pero, como veremos, tienen mucho que ver los problemas que plantean las fuentes primarias para el estudio del personaje.

\section{Aportaciones y retos en la investigación de los embarazos y partos de Leonor de Castilla}

Esa mencionada imagen "mítica" de Leonor de Castilla ha llevado a buena parte de la historiografía a sostener una visión distorsionada de su rol maternal y de la relación con sus hijos. La condición de "esposa devota" hizo creer a algunos autores —e incluso algunos aún lo mantienen hoy en día - que no había sido una madre implicada ni cariñosa ya que su esposo Eduardo I constituía el centro absoluto de su mundo ${ }^{23}$. Lejos de esa incierta e infundada idea, a pesar del poco tiempo que la reina pasó físicamente al lado de sus hijos, Parsons considera que no hay pruebas ni razones para creer que fuera una madre despreocupada, sino más bien todo lo contrario ${ }^{24}$. Este autor ve incluso pruebas de una cercanía emocional con ellos en ciertos actos, como su voluntad de retrasar el traslado de su hija Leonor a Aragón tras su matrimonio, o la tumba para el corazón de su hijo Alfonso que preparó junto a la suya en Londres ${ }^{25}$. En cualquier caso, son aún muchos los aspectos pendientes de análisis en la maternidad de Leonor de Castilla, sobre todo desde un enfoque político. Como hemos señalado, su papel como madre constituyó una de las principales fuentes de autoridad de esta reina. Sin embargo, es necesario continuar indagando acerca del modo y el grado en que las relaciones maternofiliales constituyeron para esta reina un recurso de poder, en qué medida su consolidación como madre prolífica fue paralela al afianzamiento de su posición e influencia en la corte de Inglaterra. En definitiva, el análisis de la maternidad de Leonor de Castilla desde el enfoque político tiene un

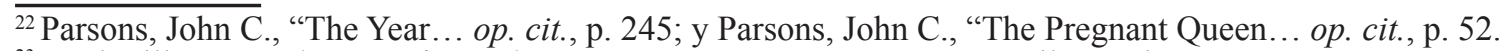

${ }^{23}$ Cockerill, Sara, Eleanor of Castile... op. cit., pp. 170, 249 y 253; y Hilton, Lisa, Queens Consort: England's Medieval Queens, Nueva York, W\&N, 2008, p. 195.

${ }^{24}$ Parsons, John C., "Legend and Reality... op. cit., p. 39; y Parsons, John C., Eleanor of Castile... op. cit., p. 39

${ }^{25}$ Para más información acerca de los hijos y otros recursos de Leonor de Castilla véase Bergaz Âlvarez, Andrea,

"Los recursos de poder de la reina Leonor de Castilla (1241-1290): un estado de la cuestión", en La España

Medieval, 44 (2021), pp. 323-347. https://doi.org/10.5209/elem.75424
} 
gran interés manifestado ya en otros estudios de caso sobre reinas que se han desarrollado en el marco de la reginalidad, y sería viable y conveniente en este caso.

La situación no es muy diferente en lo referente a la investigación sobre sus embarazos y partos. Prevalece al respecto una enorme confusión que, incluso en aspectos tan básicos como el número de hijos, continúa sin aclararse. La investigación más exhaustiva, completa y específica sobre el tema fue efectuada, una vez más, por Parsons en los años ochenta, plasmada en su artículo "The Year of Eleanor of Castile's Birth and her Children by Edward I"26. En él analizó la documentación disponible y relevante al respecto con el objetivo de aclarar el número de partos, así como la fecha y lugar de los mismos. Más adelante se han realizado algunas aportaciones, pero falta un estudio dedicado en exclusiva a esta cuestión ${ }^{27}$. En consecuencia, la información disponible se encuentra fragmentada y dispersa.

Los motivos de esta falta de atención son diversos. La escasez de fuentes primarias con datos relevantes sobre la gestación y los nacimientos tiene mucho que ver, algo que, no obstante, ocurre con la mayoría de reinas consortes. Además, tampoco contamos con testimonios materiales o monumentales, los cuales podrían ser de gran ayuda. No hay certeza del lugar ni tan siquiera de la existencia de la tumba de ninguno de los hijos de la pareja fallecidos durante la infancia, siendo este asunto en sí mismo objeto de debate. Algunos autores como Tanner o Cockerill consideran que el altar del ambulatorio sur de la catedral de Westminster es un sepulcro común de algunos de los hijos de Enrique III y Eduardo I que murieron siendo niños ${ }^{28}$. En cambio, otros como Parsons sostienen que es el altar original de la capilla de Eduardo el Confesor ${ }^{29}$. Aunque continúa sin haber consenso sobre la cuestión, la investigación arqueológica ha proporcionado nuevos indicios. El estudio de GPR (Ground Penetrating Radar) llevado a cabo en el 2005 por la Universidad de Cambridge bajo la dirección de Erica Utsi, mostró la existencia de una serie de espacios en el interior del altar que serían compatibles con enterramientos infantiles ${ }^{30}$. Más tarde, Badham publicó un artículo en el que reexaminaba la evidencia documental a la luz de los datos extraídos de este estudio arqueológico, concluyendo que "beyond doubt, a significant number of burials took place here before the 1290s", aunque admitía no poder identificar a quiénes pertenecerían cada uno de los enterramientos ${ }^{31}$.

En cuanto a las fuentes documentales, tal y como señaló Kay Staniland, es muy complicado encontrar en ellas información sobre embarazos y partos de las reinas inglesas

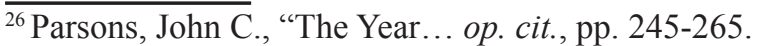

${ }^{27}$ Entre otros véase Cockerill, Sara, Eleanor of Castile... op. cit., pp. 246-267; Morris, Marc, A Great and Terrible King: Edward I and the Forging of Britain, Londres, Hutchinson, 2008, pp. 22, 73, 76-77, 101, 107, 110, 192, y 194-195; y Hilton, Lisa, Queens Consort... op. cit., pp. 190-203.

${ }^{28}$ Tanner, Joan, "Tombs of Royal Babies in Westminster Abbey", Journal of the British Archaeological Association, 16 (1953), p. 25. https://doi.org/10.1080/00681288.1953.11894721; y Cockerill, Sara, Eleanor of Castile... op. cit., pp. 158 y 192.

${ }^{29}$ Parsons, John C., "The Year... op. cit., p. 250.

${ }^{30}$ Utsi, Erica, "The Shrine of Edward the Confessor: A Study in Multi-frequency GPR Investigation", en Near Surface Geophysics, 10 (2012), sin foliar. https://doi.org/10.3997/1873-0604.2011025

${ }^{31}$ Badham, Sally, "Edward the Confessor's Chapel, Westminster Abbey: The Origins of the Royal Mausoleum and its Cosmatesque Pavement", en Antiquaries Journal, 87 (2007), pp. 197 y 213-214. https://doi.org/10.1017/ $\underline{\mathrm{S} 0003581500000895}$
} 
con anterioridad a la dinastía Tudor. Algunos documentos han permitido llevar a cabo trabajos razonablemente extensos sobre la cuestión con anterioridad a esta dinastía — por ejemplo, el de esta misma autora sobre Thomas de Brotherton-. Sin embargo, las posibilidades de estudio de reinas anteriores a Margarita de Francia (1279-1318), segunda esposa de Eduardo I, son extremadamente limitadas ${ }^{32}$. En el caso de Leonor de Castilla, la cuestión se complica aún más debido a la escasez general de fuentes documentales primarias sobre su figura. De entre los documentos de su Casa solo se conserva completo el llamado Liber Garderobe, hoja de gastos del año 1289-1290 que fue editada por Parsons y que, según Johnstone, es el documento "más sustancial" para el estudio de Leonor ${ }^{33}$. Además, han llegado a nuestros días otros dos documentos de su Casa, aunque muy fragmentados y en malas condiciones: el llamado rotulus hospicii, hoja de gastos correspondiente al año 1287-1288; y otro documento similar del año 1288-1289 prácticamente ilegible. A estas fuentes hay que sumar parte de su correspondencia se han conservado cuarenta y siete cartas, cuarenta y seis de ellas relacionadas con su actividad económica-, y el informe de la investigación llevada a cabo tras su muerte (1291-1292) con motivo de las quejas sobre su actividad patrimonial y la actuación de sus clerks (secretarios) ${ }^{34}$. Estas fuentes son muy importantes y valiosas, pero, como puede apreciarse, limitadas y escasas.

El resultado de todo ello es una enorme confusión por parte de la historiografía, que no se pone de acuerdo respecto a las fechas, lugares de nacimiento, y ni tan siquiera sobre el número total de hijos de Leonor de Castilla. Habitualmente los investigadores hablan de doce a dieciséis nacimientos, tal y como muestran los trabajos de Prestwich, Morris, Cockerill, o Parsons, entre otros ${ }^{35}$. Tampoco hay consenso acerca de la identificación de los niños que no sobrevivieron a los primeros años de vida. Algunos han sido nombrados de forma errática, lo que ha dado lugar a la aparición de hasta diecisiete nombres diferentes, muchos de ellos fruto de una identificación errónea ${ }^{36}$. Un caso paradigmático en este sentido es el de una supuesta hija llamada Alice. Desde el siglo Xv hasta el xx diversos estudiosos contribuyeron a perpetuar, mediante una cadena de errores cronológicos y de otro tipo, la existencia de esta supuesta hija de la pareja real. Fue finalmente Parsons quien desveló que, en realidad, se trataba de una confusión con el sí constatado Alfonso, que murió con once años, debido a la similitud entre el nombre de "Alice" y la traducción inglesa de este último (Amfulsus o Alfundus) ${ }^{37}$.

\footnotetext{
$\overline{{ }^{32} \mathrm{PRO}, \mathrm{E101/357}} / 20$ Issues of stuffs for the queen and Thomas of Brotherton, en Staniland, Kay, "Welcome, Royal Babe!: The Birth of Thomas of Brotherton in 1300", en Costume. The Journal of the Costume Society, 19 (1985), pp. 1-13. https://doi.org/10.1179/cos.1985.19.1.1

${ }_{33}$ Parsons, John C., Court... op. cit., pp. 3-4.

${ }^{34}$ Este informe ha sido editado y es objeto de varios trabajos. Véase Fenwick, Muriel, "The Inquiry into Complaints Against the Ministers of Eleanor of Castile, 1291-1292", M.A. thesis, University of London, 1931; y Fryde, Natalie, "A Royal Inquiry into Abuses: Queen Eleanor's Ministers in North-east Wales, 1291-92", en Welsh History Review, 5 (1970-71), pp. 366-376.

${ }_{35}^{35}$ Parsons, John C., "The Year... op. cit., p. 249; Prestwich, Michael, Edward I, New Haven, Yale University Press, 1988, p. 573; Cockerill, Sara, Eleanor of Castile... op. cit., p. 436; y Morris, Marc, A Great ... op. cit., p. 436.

${ }^{36}$ Parsons, "The Year... op. cit., p. 249.

${ }^{37}$ Sobre la confusión véanse Lane, Henry, The Royal Daughters of England, Londres, Constable and Company, 1910, pp. 198-199; y Green, Mary Anne Everett, The Lives of the Princesses of England, Londres, H. Colburn, 1849-55, pp. 404-442.
} 
Por lo tanto, la escasez y las limitaciones de las fuentes específicas sobre Leonor de Castilla plantean problemas y peligros, pues, a pesar de su relevancia, existe, tal y como advierte Cockerill, el riesgo de magnificar su importancia y producir una imagen distorsionada del personaje $\mathrm{e}^{38}$. Por ello es necesario recurrir a la información que pueden aportar fuentes más generales que no proceden de su Casa y no se refieren sólo a su persona concretamente. Entre ellas hay que incluir la correspondencia — no solamente la emanada o recibida por Leonor-, y también fuentes de carácter administrativo y económico tales como los Patent Rolls o los Liberate Rolls. Estos últimos podrían proporcionar información valiosa acerca de la vida diaria de la reina e incluso acerca de sus gustos, intereses y consumos, pues recogen los gastos llevados a cabo por la monarquía ${ }^{39}$.

Parsons ha demostrado lo útil que puede llegar a ser el cotejo de unas fuentes con otras en el estudio de los embarazos y partos, especialmente en lo referente al establecimiento de la data cronológica y geográfica. Gracias a la consulta de los Liberate Rolls pudo establecer, al menos de forma aproximada, la fecha del nacimiento del primer varón de la pareja, llamado Henry, el día 6 de mayo de $1268^{40}$. Otro documento perteneciente a la Casa del rey y a sus actividades y gastos diarios - el registro de cuentas de la Public Record Office editado por Trease - le permitió situar el nacimiento de Joan en una fecha cercana al 25 de enero de $1265^{41}$. La correspondencia privada ofrece asimismo grandes posibilidades, como bien demuestra una carta de la reina madre Leonor de Provenza a su hijo Eduardo I en la que se aclara el lugar de nacimiento de Alfonso en Bayona ${ }^{42}$. No podemos dejar de mencionar las crónicas, que en muchas ocasiones registran los nacimientos regios, si bien es cierto que, para el caso de Leonor, se limitan casi exclusivamente a los hijos varones - especialmente los herederos - o a partos difíciles. El ejemplo más claro es el nacimiento del futuro Eduardo II, establecido ya por Johnstone el 25 de abril de 1284 en Carnarvon a partir de la crónica Flores historiarum ${ }^{43}$. Siguiendo el mismo método, los Annales Monastici permitieron a Parsons localizar de forma aproximada el nacimiento de Joan de Acre en 1272, fecha que más tarde afinó Cockerill a partir del análisis de las apariciones públicas documentadas de la reina, situando el alumbramiento en mayo de ese año ${ }^{44}$.

Gracias al cotejo de estas fuentes generales, Parsons pudo llevar a cabo un análisis de los últimos meses de gestación y del parto de Mary —una de las últimas hijas de Leonor-,

\footnotetext{
${ }^{38}$ Cockerill, Sara, Eleanor of Castile... op. cit., pp. 18 y 210.

${ }^{39} \mathrm{El}$ potencial de esta fuente para los estudios sobre reginalidad inglesa -en su caso concretamente en referencia al estudio de Leonor de Provenza-, fue señalado ya por Paula del Val Vales en su comunicación "Un hogar para la reina: Las obras y edificios de Enrique III de Inglaterra para Leonor de Provenza (1236-1241)", en V Congreso de Jóvenes Investigadorxs con perspectiva de género (Universidad Carlos III de Madrid, 4 de junio de 2020). «https:// media.uc3m.es/video/5eddf9f18f420843a68b456fi [consultado el 7 de septiembre de 2021].

${ }^{40}$ Calendar of Liberate Rolls [CLR], vol. 6: 1267-1272, p. 30, no. 272, en Parsons, John C., "The Year... op. cit., p. 259.

${ }^{41}$ PRO E 101/350/1, m.1 en Trease, George, "The Spicers and Apothecaries of the Royal Household in the Reigns of Henry III, Edward I and Edward II", en Nottingham Medieval Studies, 3 (1959), p. 40. https://doi.org/10.1484/J. NMS.3.2; Parsons, John C., "The Year... op. cit., p. 258.

${ }^{42}$ PRO Ancient Correspondence, SC 1/47/109, dat. Lutgershall 14 June, en Parsons, John C., "The Year... op. cit., p. 261.

${ }^{43}$ Johnstone, Hilda, Edward of Carnarvon, Manchester, Manchester University Press, 1944, p. 6.

${ }^{44}$ Parsons, John C., "The Year... op. cit., p. 261; y Cockerill, Eleanor of Castile... op. cit., p. 176.
} 
evaluando cómo ello afectó a la vida de la reina. Demostró cómo el nacimiento de esta hija no frenó las actividades políticas de la reina, pues cinco días después del parto — que tuvo lugar el 11 o 12 de marzo de 1279 - Leonor se convirtió en condesa de Ponthieu, lo que, sin duda, la involucró en complejas maniobras administrativas y diplomáticas durante el confinamiento posparto. Constata también que, doce días después del nacimiento, la reina obtuvo la tutela de algunas tierras en East Anglia cercanas a señoríos de su propiedad, lo que generó una correspondencia que, de acuerdo con este especialista, demostraría de nuevo que Leonor se encontraba involucrada en las negociaciones, tomando parte personalmente para conseguir dichas concesiones ${ }^{45}$.

\section{Conclusión}

A lo largo de este trabajo hemos podido comprobar cómo los retos en la investigación y el estudio de la maternidad, gestación y partos de la reina de Leonor de Castilla son aún importantes. Hemos podido mostrar cómo, en gran medida, los motivos de las lagunas y de los desacuerdos de los especialistas radican en la escasez de fuentes primarias referidas al personaje, lo que plantea muchas limitaciones en su estudio. Aunque aquí hemos ofrecido solamente unos pocos ejemplos, algunas de las obras manejadas demuestran el enorme potencial que ofrecen las fuentes no específicamente referidas a Leonor de Castilla. John C. Parsons fue el primero en manejarlas sistemáticamente con tal objetivo en mente, pero lo hizo de forma limitada, pensando únicamente en datar con precisión los nacimientos de sus hijos. No obstante, también estas fuentes presentan limitaciones. Aunque han permitido en algunos casos aclarar el asunto del número de hijos de la pareja, así como el momento y lugar de los nacimientos, debemos asumir que es difícil que se puedan llegar a clarificar todas las maternidades de Leonor de Castilla. A ello hay que sumar la existencia de aspectos difícilmente historiables - aunque relevantes en el estudio de la maternidad reginal — tales como la cuestión de los abortos ${ }^{46}$.

A pesar de ello — sí merecería la pena volver a estas fuentes - tanto generales como específicas, con unas miras más amplias, a fin de evaluar sus posibilidades para la investigación de la maternidad de Leonor de Castilla. Más allá de estudios centrados en desentrañar la probablemente irresoluble —además de habitual en el caso de muchas otras reinas de la

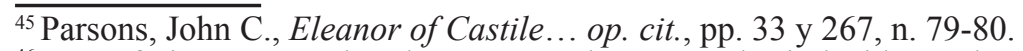

${ }^{46}$ Nos referimos tanto a los abortos naturales como a los inducidos. Sobre la cuestión existen pocas o prácticamente ninguna fuente más allá de la legislación. No obstante, la historiografía ha trabajado en ocasiones el asunto. Véanse al respecto Rodriguez Ortiz, Victoria, El aborto hasta fines de la Edad Media castellana. Su consideración social y jurídica, Pamplona, Aranzadi, 2014 y Marcos, Eduardo, "Algunas consideraciones sobre infanticidio, aborto y abandono durante la Edad Media”, en Rodríguez, Gerardo et al. (coords.), Cuestiones de historia medieval, Buenos Aires, Universidad Católica Argentina, 2010, vol. 2, pp. 155-166.
} 
época - incógnita acerca del número de nacimientos, su data cronológica y geográfica y otras cuestiones obstetricias - investigaciones que no dejan de tener un carácter positivista - , estas fuentes presentan un notable potencial para el estudio de la reginalidad de esta reina. En nuestra opinión, podrían ayudar a indagar en la mencionada importancia política que habría tenido la maternidad para Leonor de Castilla, desentrañando en qué medida la reina se valió de sus hijos y de su condición de madre para consolidar su posición, y confirmando - o no- que se trata, como Berenguela de Castilla, de un caso de "maternidad positiva". La correspondencia privada podría ser en este sentido muy interesante.

Por otro lado, un nuevo análisis actualizado de estas fuentes podría ayudar a investigar en qué medida su condición de casi permanente mujer embarazada condicionó o no su vida política como reina. Así, por ejemplo, un análisis más detenido de las mismas —ampliando a otros hijos de la pareja estudios como el realizado por Parsons del nacimiento de Mary_ podría proporcionar más información sobre los embarazos, los partos y los rituales y procedimientos asociados a ellos, permitiéndonos conocer un poco mejor cómo pudieron afectar a la actividad política de la reina y a su ejercicio práctico del poder. Especialmente destacable en este sentido es el llamado "confinamiento" que las reinas inglesas llevaban a cabo tras haber dado a luz. ${ }^{47}$.

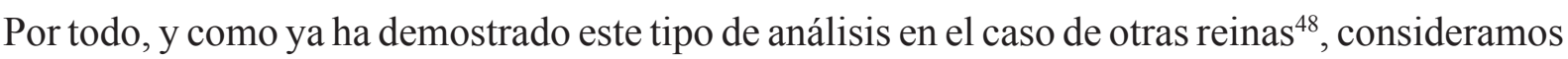
que, acompañadas de una sólida y clara base metodológica, estas fuentes ayudarían a profundizar en el conocimiento de importantes facetas de la reginalidad de Leonor de Castilla, con lo que es muy conveniente continuar indagando sus posibilidades y potencial.

\footnotetext{
${ }^{47}$ Este asunto es en sí mismo objeto de debate entre los historiadores. Parsons afirma que su duración en el caso de Leonor de Castilla era de 40 días para hijos y de 30 días para hijas ("The Year... op. cit., p. 257), algo en lo que coincide Staniland ("Welcome Royal Babe!... op. cit., p. 13). Cockerill, sin embargo, defiende una misma duración de 40 días para hijos e hijas, cumpliendo 30 sólo cuando otros asuntos apremiantes no le permitían realizar el confinamiento completo (Eleanor of Castile... op. cit., p. 249). Un análisis detallado de estas fuentes también podría también arrojar luz sobre esta cuestión.

${ }^{48}$ Por ejemplo, para Margarita de Francia, segunda esposa de Eduardo I. Véase Staniland, “Welcome Royal Babe!... op. cit., pp. 1-13.
} 